\title{
Comparison of posterior fossa volumes and clinical outcomes after decompression of Chiari malformation Type I
}

\author{
Siri Sahib S. Khalsa, MD, ${ }^{1}$ Alan Siu, MD, ${ }^{2}$ Tiffani A. DeFreitas, MS, ${ }^{4}$ Justin M. Cappuzzo, MD, ${ }^{3}$ \\ John S. Myseros, MD, ${ }^{4}$ Suresh N. Magge, MD, ${ }^{4}$ Chima O. Oluigbo, MD, ${ }^{4}$ and Robert F. Keating, MD ${ }^{4}$ \\ ${ }^{1}$ Department of Neurosurgery, University of Michigan Health System, Ann Arbor, Michigan; ${ }^{2}$ Department of Neurosurgery, George \\ Washington University; ${ }^{3}$ George Washington University School of Medicine and Health Sciences; and ${ }^{4}$ Division of Neurosurgery, \\ Children's National Health System, Washington, DC
}

OBJECTIVE Previous studies have indicated an association of Chiari malformation Type I (CM-I) and a small posterior fossa. Most of these studies have been limited by 2D quantitative methods, and more recent studies utilizing 3D methodologies are time-intensive with manual segmentation. The authors sought to develop a more automated tool to calculate the 3D posterior fossa volume, and correlate its changes after decompression with surgical outcomes.

METHODS A semiautomated segmentation program was developed, and used to compare the pre- and postoperative volumes of the posterior cranial fossa (PCF) and the CSF spaces (cisterna magna, prepontine cistern, and fourth ventricle) in a cohort of pediatric patients with $\mathrm{CM}-\mathrm{I}$. Volume changes were correlated with postoperative symptomatic improvements in headache, syrinx, tonsillar descent, cervicomedullary kinking, and overall surgical success.

RESULTS Forty-two pediatric patients were included in this study. The mean percentage increase in PCF volume was significantly greater in patients who showed clinical improvement versus no improvement in headache $(5.89 \%$ vs $1.54 \%$, $p<0.05)$ and tonsillar descent $(6.52 \%$ vs $2.57 \%, p<0.05)$. Overall clinical success was associated with a larger postoperative PCF volume increase $(p<0.05)$. These clinical improvements were also significantly associated with a larger increase in the volume of the cisterna magna $(p<0.05)$. The increase in the caudal portion of the posterior fossa volume was also larger in patients who showed improvement in syrinx $(6.63 \%$ vs $2.58 \%, p<0.05)$ and cervicomedullary kinking $(9.24 \%$ vs $3.79 \%, p<0.05)$.

CONCLUSIONS A greater increase in the postoperative PCF volume, and specifically an increase in the cisterna magna volume, was associated with a greater likelihood of clinical improvements in headache and tonsillar descent in patients with CM-I. Larger increases in the caudal portion of the posterior fossa volume were also associated with a greater likelihood of improvement in syrinx and cervicomedullary kinking.

https://thejns.org/doi/abs/10.3171/2016.11.PEDS16263

KEY WORDS Chiari malformation Type I; volumetric analysis; posterior fossa volume; outcomes

$\mathrm{C}$ HIARI malformation Type I (CM-I) is characterized by the downward displacement of the cerebellar tonsils into the foramen magnum, resulting in neurological compromise. The neurological sequelae are diverse, ranging from occipital headaches, to brainstem symptoms, to the formation of a syrinx that can itself cause myeloradiculopathic complaints. The predominant belief is that CM-I may be the result of an inherently smaller posterior fossa, which predisposes to the overcrowding and subsequent herniation of the cerebellar tonsils into the upper cervical spine, resulting in altered CSF flow dynamics. This is in contrast to Chiari malformations that are the result of obstructed fourth ventricle outflow, increased supratentorial intracranial pressure, or skull-base instabilities in the setting of hypermobility syndromes such as Ehlers-Danlos.

A multitude of studies have attempted to define the constituents of a small posterior fossa through linear measurements and morphometrics in 2D planes. Although limited, these studies were able to establish an association of CM-I with a smaller posterior fossa compared with normal controls. ${ }^{4-6,9,11,13,18}$ However, the specific parameters used to estimate the size of the posterior fossa in these studies varied (such as occipital bone hypoplasia and foramen magnum dimension), and also included rigorous measurements that 
hinder direct clinical application. More current studies have used 3D volumetric analyses to confirm reduced posterior fossa volumes in CM-I with improvements after decompression, ${ }^{1,10,12,16,19}$ but these techniques required time-consuming manual segmentation, perhaps limiting routine clinical application. Recently, Bagci et al. demonstrated a method for automated segmentation of posterior fossa MR images using a reference atlas-based method in a small cohort. ${ }^{2}$ Interestingly, they found that none of the linear landmark measurements previously used to infer posterior fossa size correlated with the true volume, indicating that further studies are necessary to validate the utility of posterior fossa volumetrics.

In this study, we sought to answer the clinical question of whether larger volume changes of the posterior fossa after surgical decompression are associated with greater likelihood of clinical improvements. To answer this question, we aimed to develop a semiautomated 3D volume segmentation program that can be applied to both preoperative and postoperative anatomy. The goal was to efficiently quantify the preoperative and postoperative $3 \mathrm{D}$ volumes of the posterior cranial fossa (PCF) using brain MR images for a cohort of pediatric patients with CM-I. These volume changes of the posterior fossa after suboccipital decompression were compared with improvements in clinical and radiographic outcomes.

\section{Method}

\section{Algorithm Overview}

The computer program developed for this study allows for accelerated rigorous 3D segmentation of the complete volume of the PCF. The authors developed this tool using MATLAB. The user interacts with the program via a graphical user interface (Fig. 1). The axial T2-weighted images from the patient's brain MRI are loaded into the program. The program then assists the user in segmenting the PCF using automated edge detection, to calculate the volume of the PCF. After the posterior fossa volume has been segmented, CSF subvolumes are detected within regions of interest using an intensity threshold with noise filtering. The program also allows for 3D visualization of the segmented volume, visual comparison of two different volumes (e.g., preoperative vs postoperative volumes), and a superimposed CSF subvolume on the segmented posterior fossa, as detailed and illustrated below.

\section{Posterior Fossa Segmentation Algorithm}

The process used by the MRI segmentation tool is depicted by the flowchart shown in Fig. 2. Axial MRI slices of the patient's head, read as DICOM files, are loaded into the program. These axial slices are used to construct a midsagittal view to allow the user to define the midsagittal

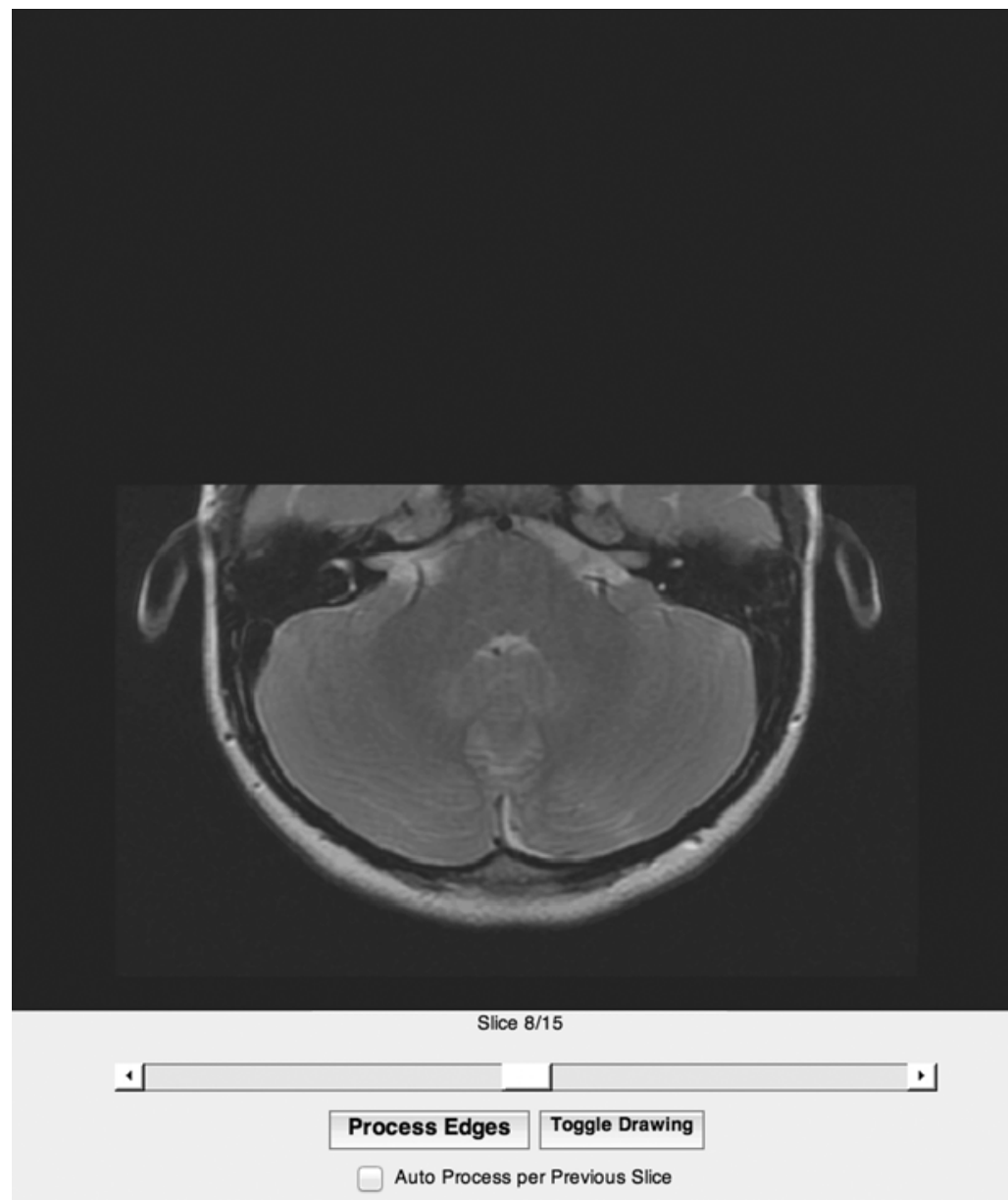

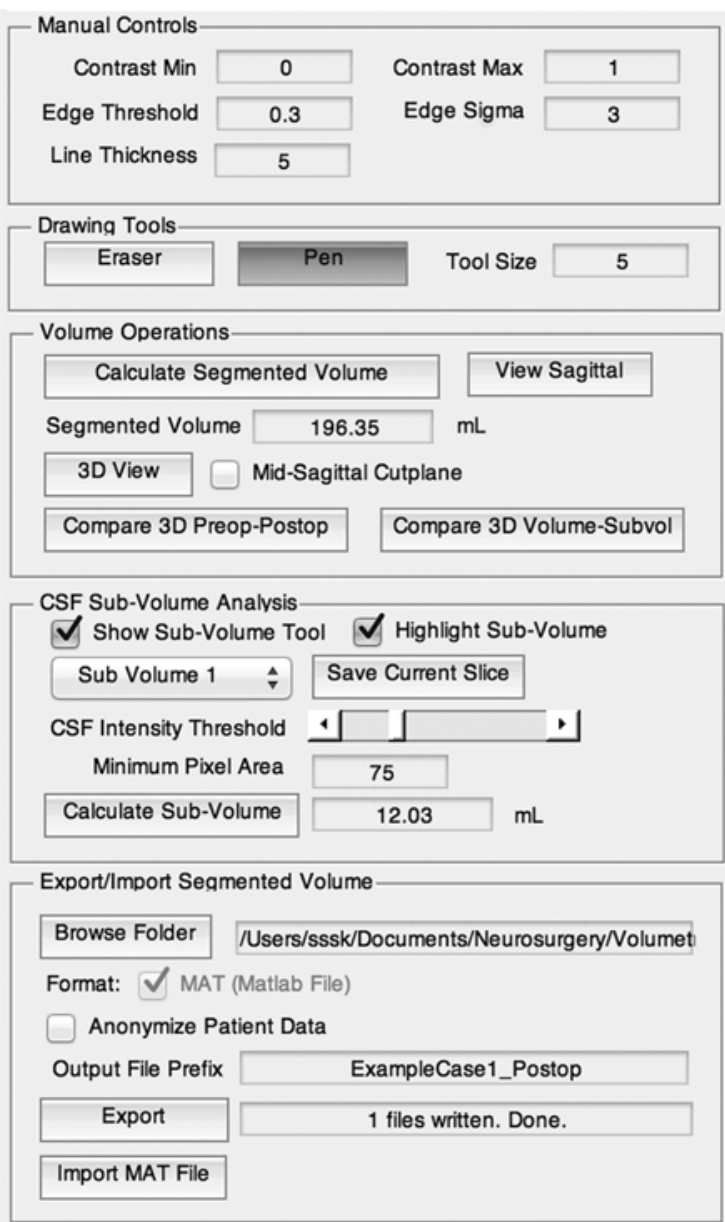

FIG. 1. Graphical user interface of the segmentation tool developed for this project. 


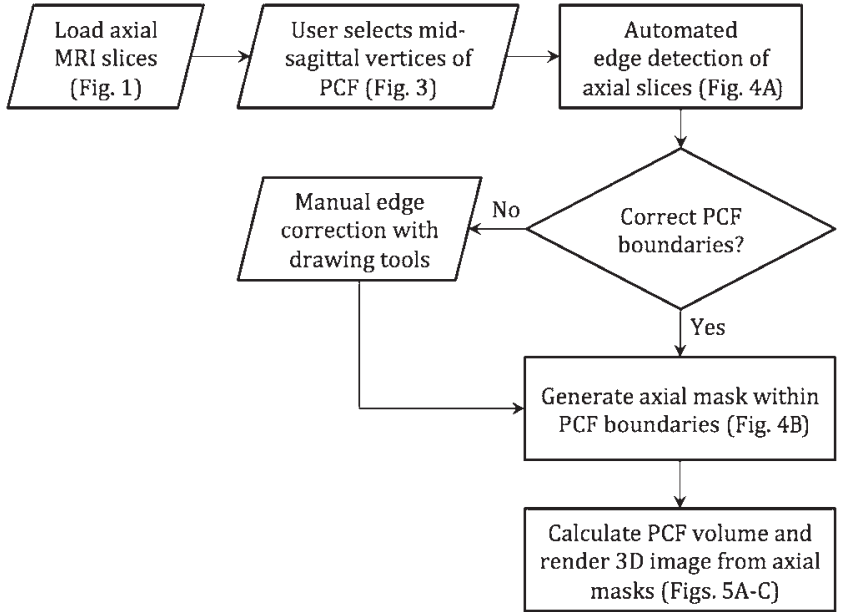

FIG. 2. Flowchart depicting the PCF segmentation process using the segmentation tool.

boundaries of the PCF. The polygon defined by the user-selected vertices sets the cranial-caudal limits of the PCF as well as the unique anterior-posterior limits for each axial slice. This step is necessary to define the sloped border extending from the tentorium to the clivus (points shown in Fig. 3), which is not visible on axial slices and thus prevents traditional edge detection. The program then performs automated edge detection on each axial slice. The initial edge detection is performed by locating local maxima in the intensity gradient on an image smoothed with a gaussian filter, as described by Canny. ${ }^{3}$ The edges are then displayed to the user (Fig. 4A), who chooses the boundary of interest by clicking once with the mouse within the desired area. If the boundaries of the PCF are not correctly identified for a given axial slice by the automated process, the user can enable a drawing tool to manually correct the edges. The program then automatically fills the region of interest to create a binary mask corresponding to the PCF contents



FIG. 3. Sagittal T2-weighted MR image showing the 2 most important points for the user to define on the midsagittal reconstruction: the superior tips of the clivus (1) and tentorium (2). The caudal posterior fossa was defined as the volume of the posterior fossa inferior to a line drawn between the cranial tip of the clivus anteriorly and the torcula posteriorly (dashed line). Figure is available in color online only.
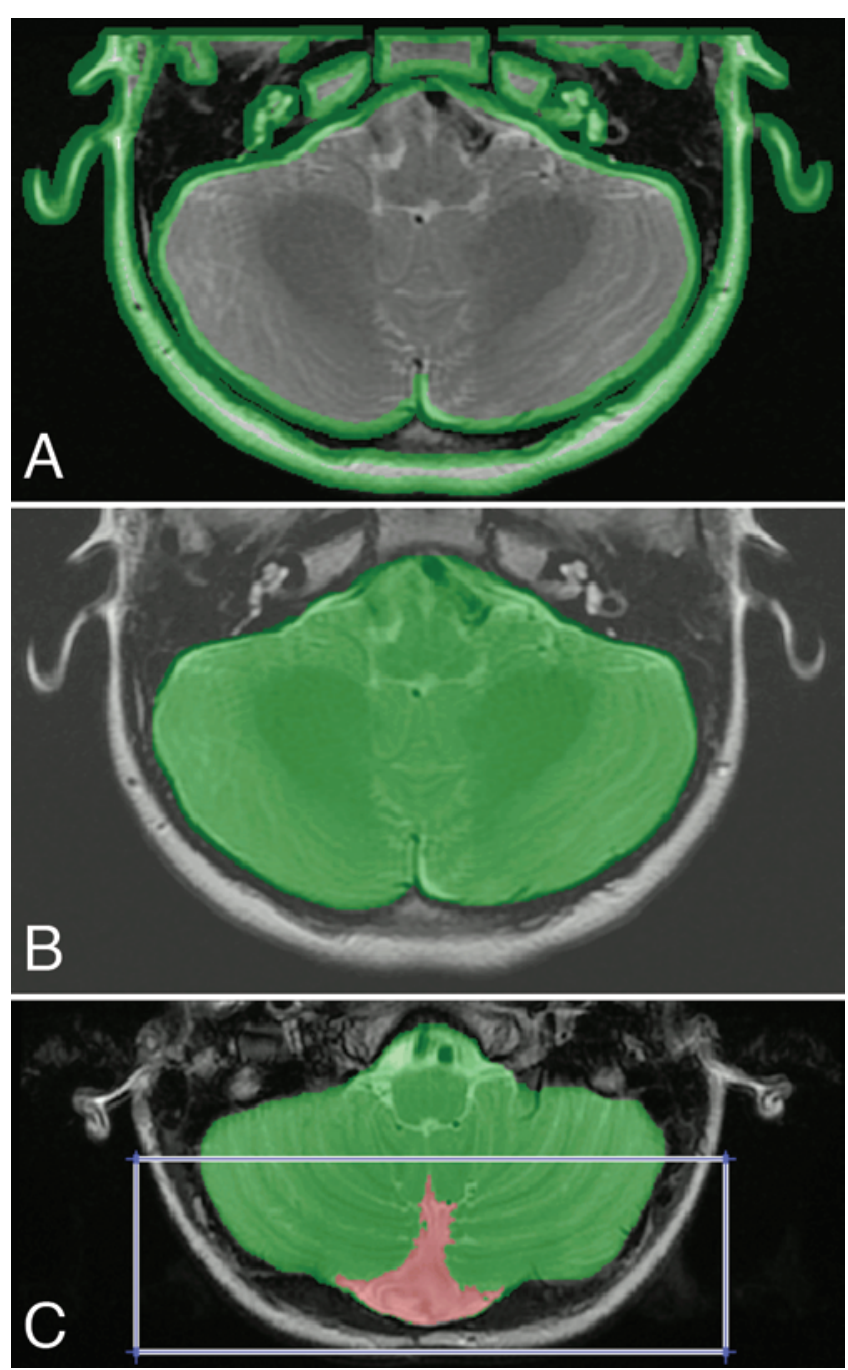

FIG. 4. A: Edges automatically generated by the segmentation tool. B: After the user clicks within the desired boundary, the contained pixels are highlighted and stored in a binary matrix. C: CSF subvolume analysis. The volume of the CSF cistern of interest (red) was automatically calculated after semiautomated segmentation of the posterior fossa (green). Figure is available in color online only.

(Fig. 4B). This process is repeated for each axial slice within the previously defined cranial-caudal limits of the PCF. The final segmented volume is stored as a 3D binary matrix. The total processing time is approximately 5 minutes for an MR image with 5-mm spacing between axial slices.

\section{CSF Subvolume Segmentation Algorithm}

After the PCF volume is segmented, the program is ready to segment CSF subvolumes. The user draws a polygon to define the region of interest that contains the desired CSF space (Fig. 4C). The program takes advantage of the hyperintensity of CSF on the T2-weighted sequence by highlighting only those pixels above a minimum intensity threshold that are contained within the user-specified region of interest. Noise is filtered by removing all highlighted areas with fewer than a user-specified minimum number of connected pixels, which are counted via the 8-pixel connected neighborhood around each highlighted pixel. 


\section{Volume Calculation and 3D Rendering}

The volume of the segmented PCF can be calculated by clicking a button within the graphical user interface of the MRI segmentation tool. The program calculates the volume of the PCF in milliliters using the following equation:

$$
\begin{aligned}
& \text { Volume }=(\text { Spacing Between Slices }) \\
& \quad(\text { Pixel Spacing })_{x} \cdot(\text { Pixel Spacing })_{y} \cdot \sum_{x, y, z} I(x, y, z)
\end{aligned}
$$

in which "Spacing Between Slices" and "Pixel Spacing" refer to the cranial-caudal and transverse lengths, respectively, of each voxel in millimeters as defined in the metadata embedded in the MRI DICOM files. The summation over all " $I(x, y, z)$ " equals the number of pixels contained within the entire PCF volume on MRI. The program can also generate a 3D rendered image of the segmented volume, which the user can rotate to various views (Fig. 5A). In addition, two different volumes can be visually compared in a color-coded 3D view (Fig. 5B). The program uses $3 \mathrm{D}$ gridded interpolation to allow for the comparison of two volumes generated from MRI with nonequal resolutions, as may be the case when preoperative and postoperative MRI are captured at different hospitals. In addition, 3D renderings of the CSF subvolumes can be overlaid on the complete PCF volume (Fig. 5C).

\section{Study Design}

This study was conducted retrospectively at Children's National Health System, in Washington, DC. Approval was obtained by the institutional review board. Informed consent was obtained from participants, or legal guardians in the case of underage or incapacitated participants. Inclusion criteria were children 21 years old and under with CM-I who underwent surgical decompression between 2005 and 2013. Patients without both preoperative and postoperative axial T2-weighted MRI of complete PCF were excluded from the study due to an inability to perform the necessary calculations. The clinical and radiographic outcomes monitored were back pain, extremity symptoms, gait instability, headache, hoarseness, hyperreflexia, oculomotor symptoms, scoliosis, sleep apnea, snoring, swallowing difficulties, surgical success/failure, basilar invagination, cervicomedullary kinking, syrinx, and tonsillar descent. Surgical success was defined as any improvement in symptoms. Surgical failure was defined as the need for repeat decompression. After patients were selected for analysis, their preoperative and postoperative MR images were analyzed using our custom software described above. The operator of the software was blinded to the clinical outcomes while performing the segmentation and volume calculation.

\section{Results}

The posterior fossa volume increase after suboccipital decompression was calculated for a total of 42 patients with CM-I (29 males, 13 females). The numbers of patients improved versus those not improved for each clinical outcome and surgery type are listed in Table 1 . The mean age at the time of surgery was $7.2 \pm 4.5$ years. The
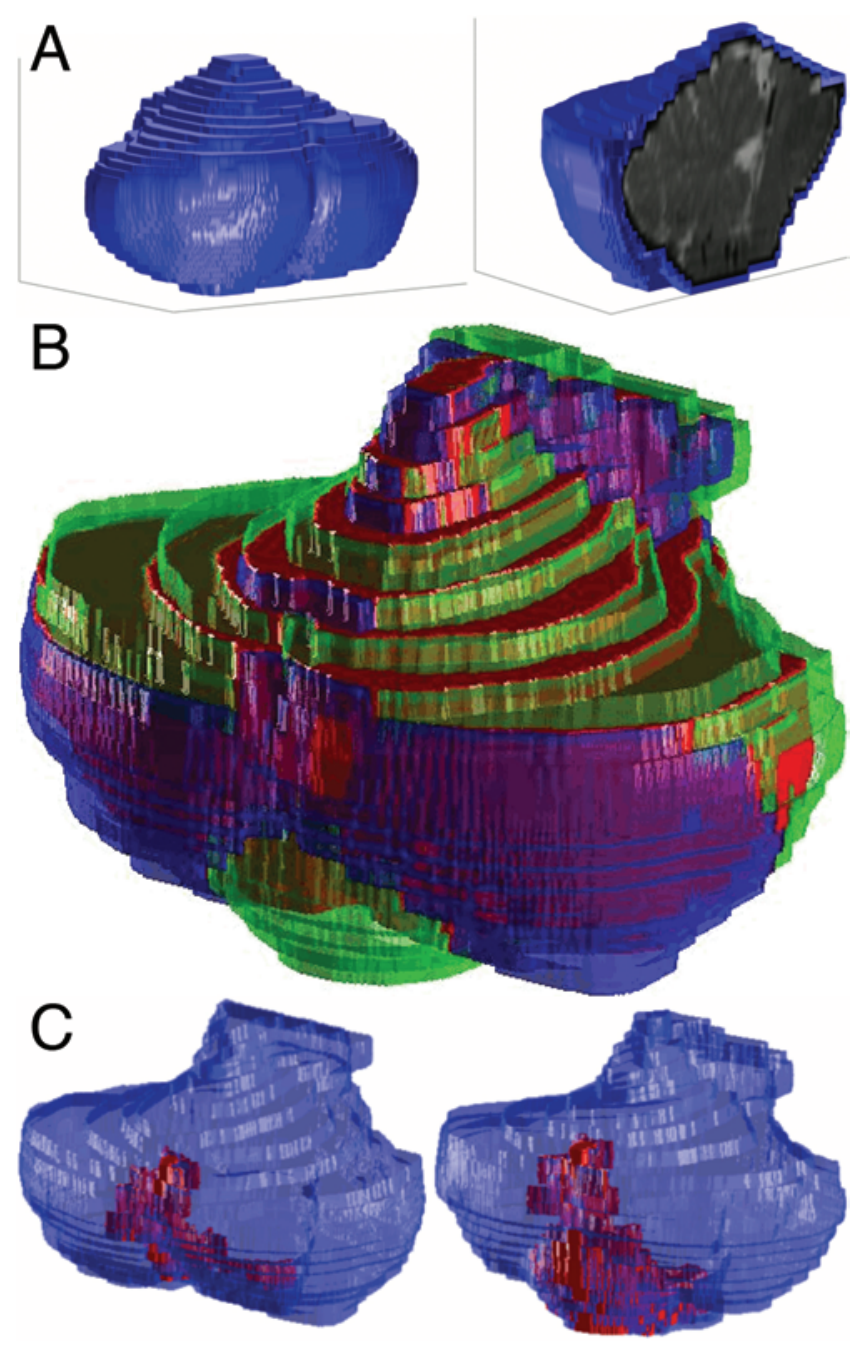

FIG. 5. A: Three-dimensional rendering of the PCF segmented by the MRI segmentation tool (left), and midsagittal cut-plane within the 3D rendering (right). B: Three-dimensional overlap (red) of the preoperative (blue) and postoperative (green) volumes of the PCF in a patient with $\mathrm{CM}$-I who underwent suboccipital decompression. Note the enlarged cisterna magna in the postoperative volume. C: Example of 3D comparison of cisterna magna volumes (red) preoperatively (left) versus postoperatively (right) using the segmentation tool developed for this project. Figure is available in color online only.

mean follow-up duration to determine symptom resolution was $2.2 \pm 1.7$ years. Preoperative posterior fossa volumes ranged from 102 to $241 \mathrm{ml}$, with a mean of $182 \mathrm{ml}$. The mean posterior fossa volume increase for all participants was $9.3 \pm 10.8 \mathrm{ml}$. A syrinx was present in $23(54.8 \%)$ of 42 patients. Before selection of the 5 clinical outcomes that would be used for analysis, the number of patients who improved versus failed to improve in each of the original 16 monitored outcomes was counted. The surgical success, tonsillar descent, cervicomedullary kinking, syrinx, and headache outcomes were the only outcomes with a sufficient sample size of patients in both the improvement and no-improvement groups and were thus chosen for analysis. Hypothesis testing was completed using unequal-variance t-tests for continuous variables, and the chi-square test for categorical variables. 
TABLE 1. Number of patients improved versus not improved for each clinical outcome and surgery type

\begin{tabular}{lccr}
\hline \multirow{2}{*}{ Clinical Outcome } & \multicolumn{3}{c}{$\begin{array}{c}\text { No. Improved/Not Improved } \\
\text { (success rate \%) }\end{array}$} \\
\cline { 2 - 4 } & Duraplasty & Bone-Only & Total \\
\hline Headache & $13 / 1(92.9)$ & $11 / 3(78.6)$ & $24 / 4(85.7)$ \\
\hline Syrinx & $11 / 4(73.3)$ & $4 / 4(50)$ & $15 / 8(65.2)$ \\
\hline Tonsillar descent & $16 / 3(84.2)$ & $12 / 5(70.6)$ & $28 / 8(77.8)$ \\
\hline Cervicomedullary kinking & $4 / 3(57.1)$ & $1 / 5(16.7)$ & $5 / 8(38.5)$ \\
\hline Surgical outcome & $19 / 3(86.4)$ & $17 / 3(85)$ & $36 / 6(85.7)$ \\
\hline
\end{tabular}

The mean posterior fossa volume change after surgery, as calculated by our segmentation tool, was compared between patients who improved versus those who did not improve for the outcomes of headache, syrinx, tonsillar descent, cervicomedullary kinking, and surgical outcome (Fig. 6A). The mean posterior fossa volume increase after surgery for patients with versus without improvement in clinical outcomes was $5.89 \%$ versus $1.54 \%$ for headache ( $p$ $=0.013), 4.39 \%$ versus $3.63 \%$ for syrinx $(\mathrm{p}=0.32), 6.52 \%$ versus $2.57 \%$ for tonsillar descent $(\mathrm{p}=0.025), 4.98 \%$ vs. $2.47 \%$ for cervicomedullary kinking $(\mathrm{p}=0.16)$, and $5.86 \%$ versus $3.08 \%$ for surgical outcome $(p=0.031)$.

A subvolume analysis revealed that the volume change of the cisterna magna revealed a similar pattern as the analysis of overall posterior fossa volume (Fig. 6B), again showing a statistically significant difference in volume change affecting headache $(\mathrm{p}=0.036)$, tonsillar descent ( $\mathrm{p}$ $=0.0049)$, and surgical outcome $(p=0.0061)$. The relative volume change of a CSF cistern was defined as the ratio of the CSF volume change after surgery to the preoperative CSF volume.

We also analyzed outcomes comparing changes in only the caudal portion of the posterior fossa, with the hypothesis that the volume increases in the caudal region of the posterior fossa would serve a more important role in affecting clinical outcomes (Fig. 6C). The caudal posterior fossa was defined as the volume of the posterior fossa inferior to a line drawn between the cranial tip of the clivus anteriorly and the torcula posteriorly. The mean caudal posterior fossa volume increase after surgery for patients with versus without improvement in clinical outcomes was $7.74 \%$ versus $2.26 \%$ for headache $(\mathrm{p}=0.015), 6.63 \%$ versus $2.58 \%$ for syrinx $(p=0.020), 8.21 \%$ versus $2.44 \%$ for tonsillar descent $(p=0.0038), 9.24 \%$ versus $3.79 \%$ for cervicomedullary kinking $(\mathrm{p}=0.010)$, and $7.43 \%$ versus $2.97 \%$ for surgical outcome $(\mathrm{p}=0.023)$.

Regarding surgical technique, $52 \%$ involved a duraplasty, whereas $48 \%$ underwent a "bone-only" technique of craniectomy plus removal of only the outer dural leaf. Volume changes in the total posterior fossa and cisterna magna were compared between the duraplasty and boneonly groups (Fig. 7), showing a significantly larger volume increase associated with duraplasty $(\mathrm{p}=0.015$ for total posterior fossa volume, $p=0.0028$ for cisterna magna). Patients were more likely to improve in all clinical outcomes in the duraplasty group, but this result was not statistically significant (Table 1, Fig. 8).
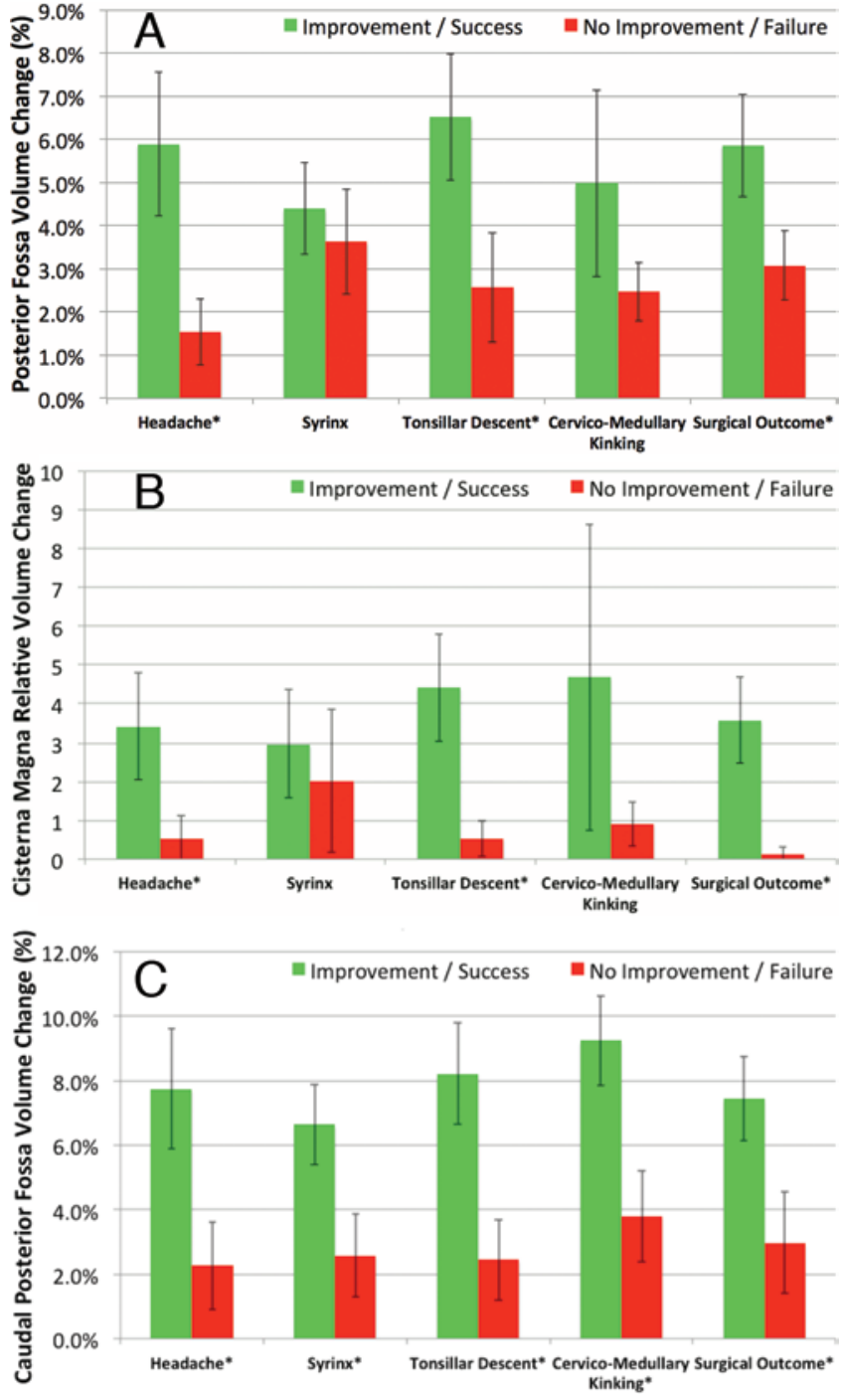

FIG. 6. Bar graphs showing comparisons between patients who improved and those who did not improve for each outcome for the following variables: mean posterior fossa volume change $(A)$, mean relative CSF volume change of the cisterna magna $(B)$, and mean volume change using only the caudal portion of the posterior fossa (C). Error bars are standard error of the mean. ${ }^{*} p<0.05$. Figure is available in color online only.

\section{Discussion}

The operative goal in the treatment of a symptomatic $\mathrm{CM}-\mathrm{I}$ is to increase the volume of the posterior fossa and restore flow of CSF across the craniocervical junction.? The goal of this study was to determine whether the magnitude of posterior fossa volume change after surgical decompression is associated with clinical outcomes in children with CM-I. Previous studies that have quantified posterior fossa volumes from MRI have used 2D volume surrogate measurements ${ }^{15}$ or time-intensive 3D manual segmentation methods..$^{14}$ Grid-based volume estimation techniques have also been used. ${ }^{17}$ Atlas-based automated segmentation techniques ${ }^{2}$ have been used to calculate the posterior fossa volume using the academic software FSL, ${ }^{8}$ but this technique has not been applied to postoperative anatomy. 


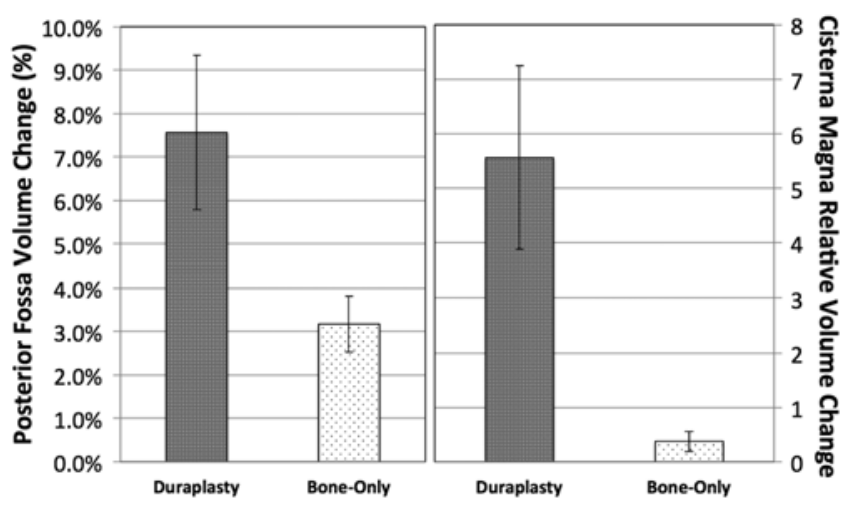

FIG. 7. Comparison of volume changes after surgery, between duraplasty and bone-only techniques, using first available postoperative MRI. Left: Total posterior fossa $(p<0.05)$. Right: Cisterna magna $(p<0.05)$.

To calculate the preoperative and postoperative PCF volumes necessary to answer our research question, we developed a semiautomated computer program that rigorously calculates the 3D posterior fossa volume from T2-weighted brain MRI. Our technique used automated edge detection. Edge detection techniques are already available using the National Institutes of Health's software ImageJ in addition to various commercial tools, and they have been effective in automated tumor volumetrics. However, the PCF poses a unique challenge that prevents complete segmentation by traditional edge detection methods. Specifically, the axial slices superior to the clivus and inferior to the tentorium lack an anterior edge. The algorithm developed for this study addressed this limitation by transforming landmarks on the midsagittal plane into axial boundary lines prior to edge detection.

In our study, a custom program was used to compare the relative posterior fossa volume increase after surgery between patients with and without successful improvement in clinical outcomes. Postoperative improvement in headache, tonsillar descent, and overall surgical outcome were found to be significantly associated with a larger postoperative volume increase in the PCF after surgery. This supports the understanding that a small and overcrowded PCF may contribute to the development of symptoms in patients with CM-I. The mean posterior fossa volume increase in patients who saw improvement in syrinx and cervicomedullary kinking was also larger than in those patients who failed to improve, but the results were not statistically significant for these two outcomes.

In addition, a subvolume analysis that isolated the 3D volume calculation for CSF showed a statistically significant difference in volume change affecting headache, tonsillar descent, and surgical outcome, which is the same pattern of results calculated for overall posterior fossa volume change. This also suggests that the important volume change that affects clinical outcomes occurs at the craniocervical junction, which is the expected result based on the theory that alteration of normal CSF flow dynamics across the foramen magnum is involved in symptom development in CM-I. Consistent with this finding, the volume changes of the prepontine cistern and the fourth ventricle did not significantly affect clinical outcomes.

When the caudal portion of the posterior fossa was iso-

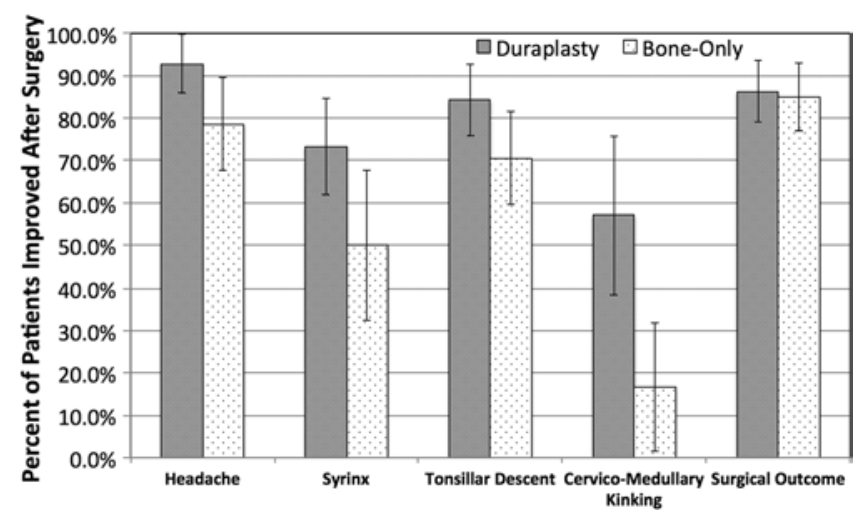

FIG. 8. Comparison of clinical outcomes between duraplasty and boneonly techniques. Differences shown are not statistically significant.

lated for volumetric analysis, all clinical outcomes (headache, syrinx, tonsillar descent, cervicomedullary kinking, and surgical outcome) were found to be significantly associated with a larger postoperative volume increase after surgery. This statistically significant improvement was expected, as this technique isolated only the portion of the posterior fossa involved in decompression surgery.

Confounding factors affect the lack of statistically significant differences in outcomes between duraplasty and bone-only surgery, in spite of the significant difference in volume change between these techniques. The postoperative MRI used for volume calculations was obtained a mean of 8 months after surgery, whereas the mean duration of follow-up for determining symptom resolution was 26 months after surgery. The bone-only patients may have undergone additional dural expansion between these 8- and 26-month mean time periods. In addition, the duraplasty and bone-only groups were not randomized. The decision to proceed with duraplasty was based on intraoperative ultrasonography used to estimate the adequacy of decompression after removal of the bone flap and outer dural leaf.

There were limitations to both the semiautomated 3D volume segmentation program and the study design. The automated edge detection methods used were successful and efficient for the majority of the PCF volume, particularly against the high-contrast borders of bone. However, automated edge detection tended to be less accurate in the low-contrast borders of the superior aspect of the PCF, where the temporal lobe and cerebellum are in close proximity; in such instances, corrective manual segmentation was used to preserve accuracy but with the consequence of increasing segmentation time. In addition, our study was designed as a retrospective analysis of clinical outcomes, which allowed us to identify an association between a larger posterior fossa volume increase after surgery and greater likelihood of clinical improvements. However, prospective studies will need to be performed to make conclusions on the ideal magnitude of posterior fossa volume increases to maximize the likelihood of improved clinical outcomes. In addition, it would be worthwhile to include CSF flow metrics as part of our database as well as compare this to our 3D volumetric analysis. We have a large prospective study in place and hope to include 3D posterior fossa data as well as CSF flow velocities in the future. 
Validation of this technique was a precursor to eventual inclusion of this valuable information. Additionally, we plan on eventually using this tool to assess whether any of our more than 600 asymptomatic patients with incidentally discovered CM-I that we follow are likely to develop future symptoms/neurological signs or syringomyelia.

\section{Conclusions}

A statistically significant association was found between a larger increase in the total posterior fossa volume and the cisterna magna CSF volume after CM-I decompression and improvement in headache, tonsillar descent, and surgical outcome. When the caudal portion of the posterior fossa volume was isolated, larger volume increases were associated with statistically significant increases in syrinx and cervicomedullary kinking in addition to headache, tonsillar descent, and surgical outcome. Decompression volumes will need to be correlated with clinical outcomes in a prospective study before conclusions can be made on the optimal decompression size or technique. The 3D volumes necessary for this analysis were calculated from brain MRI using a custom semiautomated computer program developed for this project.

\section{Acknowledgments}

Research funding for this study was provided by the American Syringomyelia \& Chiari Alliance Project.

\section{References}

1. Badie B, Mendoza D, Batzdorf U: Posterior fossa volume and response to suboccipital decompression in patients with Chiari I malformation. Neurosurgery 37:214-218, 1995

2. Bagci AM, Lee SH, Nagornaya N, Green BA, Alperin N: Automated posterior cranial fossa volumetry by MRI: applications to Chiari malformation type I. AJNR Am J Neuroradiol 34:1758-1763, 2013

3. Canny J: A computational approach to edge detection. IEEE Trans Pattern Anal Mach Intell 8:679-698, 1986

4. Dagtekin A, Avci E, Kara E, Uzmansel D, Dagtekin O, Koseoglu A, et al: Posterior cranial fossa morphometry in symptomatic adult Chiari I malformation patients: comparative clinical and anatomical study. Clin Neurol Neurosurg 113:399-403, 2011

5. Furtado SV, Reddy K, Hegde AS: Posterior fossa morphometry in symptomatic pediatric and adult Chiari I malformation. J Clin Neurosci 16:1449-1454, 2009

6. Furtado SV, Thakre DJ, Venkatesh PK, Reddy K, Hegde AS: Morphometric analysis of foramen magnum dimensions and intracranial volume in pediatric Chiari I malformation. Acta Neurochir (Wien) 152:221-227, 2010

7. Heiss J, Oldfield EH: Management of Chiari malformations and syringomyelia, in Quiñones-Hinojosa A (ed): Schmidek and Sweet's Operative Neurosurgical Techniques, ed 6. Philadelphia: Saunders, 2012, pp 2071-2080

8. Jenkinson M, Beckmann CF, Behrens TE, Woolrich MW, Smith SM: Fsl. Neuroimage 62:782-790, 2012

9. Karagöz F, Izgi N, Kapíjcíjoğlu Sencer S: Morphometric measurements of the cranium in patients with Chiari type I malformation and comparison with the normal population. Acta Neurochir (Wien) 144:165-171, 2002

10. Milhorat TH, Chou MW, Trinidad EM, Kula RW, Mandell $\mathrm{M}$, Wolpert C, et al: Chiari I malformation redefined: clinical and radiographic findings for 364 symptomatic patients. Neurosurgery 44:1005-1017, 1999

11. Nishikawa M, Sakamoto H, Hakuba A, Nakanishi N, Inoue
Y: Pathogenesis of Chiari malformation: a morphometric study of the posterior cranial fossa. J Neurosurg 86:40-47, 1997

12. Noudel R, Gomis P, Sotoares G, Bazin A, Pierot L, Pruvo JP, et al: Posterior fossa volume increase after surgery for Chiari malformation Type I: a quantitative assessment using magnetic resonance imaging and correlations with the treatment response. J Neurosurg 115:647-658, 2011

13. Noudel R, Jovenin N, Eap C, Scherpereel B, Pierot L, Rousseaux P: Incidence of basioccipital hypoplasia in Chiari malformation type I: comparative morphometric study of the posterior cranial fossa. Clinical article. J Neurosurg 111:1046-1052, 2009

14. Sgouros S, Kountouri M, Natarajan K: Posterior fossa volume in children with Chiari malformation Type I. J Neurosurg 105 (2 Suppl):101-106, 2006

15. Stovner LJ, Bergan U, Nilsen G, Sjaastad O: Posterior cranial fossa dimensions in the Chiari I malformation: relation to pathogenesis and clinical presentation. Neuroradiology 35:113-118, 1993

16. Trigylidas T, Baronia B, Vassilyadi M, Ventureyra EC: Posterior fossa dimension and volume estimates in pediatric patients with Chiari I malformations. Childs Nerv Syst 24:329-336, 2008

17. Tubbs RS, Wellons JC III, Smyth MD, Bartolucci AA, Blount JP, Oakes WJ, et al: Children with growth hormone deficiency and Chiari I malformation: a morphometric analysis of the posterior cranial fossa. Pediatr Neurosurg 38:324-328, 2003

18. Urbizu A, Poca MA, Vidal X, Rovira A, Sahuquillo J, Macaya A: MRI-based morphometric analysis of posterior cranial fossa in the diagnosis of Chiari malformation Type I. J Neuroimaging 24:250-256, 2014

19. Vurdem UE, Acer N, Ertekin T, Savranlar A, Inci MF: Analysis of the volumes of the posterior cranial fossa, cerebellum, and herniated tonsils using the stereological methods in patients with Chiari Type I malformation. Sci World J 2012:616934, 2012

\section{Disclosures}

The authors report no conflict of interest concerning the materials or methods used in this study or the findings specified in this paper.

\section{Author Contributions}

Conception and design: Keating, Khalsa, Siu. Acquisition of data: Khalsa, Siu, DeFreitas, Myseros, Magge, Oluigbo. Analysis and interpretation of data: Keating, Khalsa, Siu. Drafting the article: Khalsa, Siu, Cappuzzo. Critically revising the article: Keating, Khalsa, Siu, Cappuzzo. Reviewed submitted version of manuscript: Keating, Khalsa, Siu. Approved the final version of the manuscript on behalf of all authors: Keating. Statistical analysis: Khalsa, Siu. Administrative/technical/material support: DeFreitas. Study supervision: Keating. Operating surgeon: Keating, Myseros, Magge, Oluigbo. Wrote the code for the computer program: Khalsa.

\section{Supplemental Information}

\section{Previous Presentations}

Portions of this work were presented as an oral presentation at the 2016 AANS Annual Meeting in Chicago, Illinois, April 30May 4.

\section{Correspondence}

Robert F. Keating, Division of Neurosurgery, Children's National Health System, 111 Michigan Ave. NW, Washington, DC 20010. email: rkeating@childrensnational.org. 